\title{
A Multi-Objective Ant Colony System Algorithm for Virtual Machine Placement
}

\author{
Renu Saini, Nishant Anand \\ CBS Group of Institutions, Jhajjhar, Delhi (NCR)
}

\begin{abstract}
Virtual machine placement is a process of mapping virtual machines to physical machines. The optimal placement is important for improving power efficiency and resource utilization in a cloud computing environment. In this paper, we propose a multi-objective ant colony system algorith $\mathrm{m}$ for the virtual machine placement problem. The goal is to efficiently obtain a set of non-dominated solutions (the Pareto set) that simultaneously minimize total resource wastage and power consumption. The proposed algorithm is tested with some instances from the literature. Its solution performance is compared to that of an existing algorith $\mathrm{m}$. The results show that the proposed algorithm is more efficient and effective than the methods we compared it to.
\end{abstract}

\section{INTRODUCTION}

In the case of ACO algorithms the theoretical analyses of their runtime behavior has been started only recently. We increase the theoretical understanding of ACO algorithms by investigating their runtime behavior on the wellknown traveling salesperson (TSP) problem. For ACO the TSP problem is the first problem where this kind of algorithms has been applied. Therefore, it seems to be natural to study the behavior of ACO algorithms for the TSP problem from a theoretical point of view in a rigorous manner. ACO algorithms are inspired by the behavior of ants to search for a shortest path between their nest and a common source of food. It has been observed that ants find such a path very quickly by using indirect communication via pheromones. This observed behavior is put into an algorithmic framework by considering artificial ants that construct solutions for a given problem by carry ing out random walks on a so-called construction graph. The random walk (and the resulting solution) depends on pheromone values that are values on the edges of the construction graph.

\section{ANT COLONY SYSTEM}

Ant is a small insect that live in colonies in/on the ground. Ants explore their area surrounding the nest for food in random manner. While searching the food, ant leaves chemical materials called pheromone. Ant can smell these pheromones. While choosing the path, they tend to choose the path which contains strong pheromones concentration and this path chosen by ants on the bases of probability. When an ant finds the food source, it evaluates the food quality and quantity and carries some food with it and return to its nest. During coming back to the nest, the quantity of pheromones it leaves on the ground is depends on the quality and quantity of food. Pheromones trials guide other ant to find the food source. The communication of the ants via pheromones trail finds the shortest path between the nest and food source.

Ant colony optimization algorith $\mathrm{m}$ is now used to solve the numerous optimization problems like TSP (Traveling Salesman Problem), FSSP (Flow Shop Scheduling Problem) etc. some extension of ACO algorithm proposed in literature like Ant System, ACS and MMAS etc.

The system works as: in initialization phase, all parameters are initialized and pheromones trial is set to $\tau_{0}$. In next Iterative part each ant receives the request from all the VMs and then assigning VMs to physical server. Assigning the VM.s to physical server is based on pseudorandom proportional rule which is used by ant to choose the particular VM as the next one to fit into the current physical server. This rule is made on the bases of the information of current pheromone concentration level while movement of ant and this guide the ant to choose the most appropriate VM. There are two updates are performed. First is local update. Local update is performed once an ant has built movement. Second is global update. Global update is performed with current Pareto set when ants constructed their solutions.

There are 3 point which differ the algorith $m$ s are:

\section{Pheromone updates:}

There are two strategies; first strategy is to select the iteration-best, or a best-so-far solution to update the pheromone matrices, second strategy is to collect and store the non-dominated solutions in an external set. Only the solutions in the nondominated set are allowed to update the pheromones. 
2. Definition of pheromones and heuristic information:

There are two approaches to define heuristic/pheromones information first is using one matrix and second is multiple matrixes.

\section{Pheromone and heuristic aggregation:}

When multiple matrices are used then some aggregation rule should be used to combine mu ltip le phero mones matrices

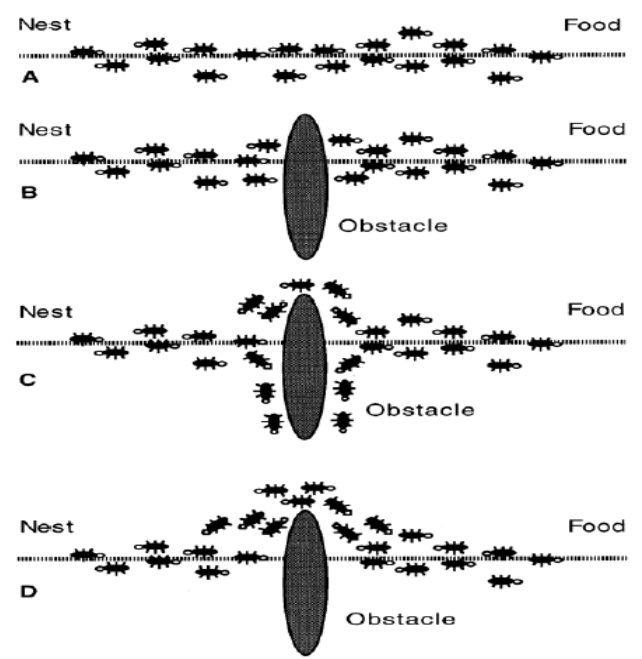

Fig.1: how real ant finds the shortest path

\subsubsection{MMAS (Min-Max Ant System):}

MMAS is also based on the ant colony system. It based on the search paradigm that is applicable for the solution of optimization problem. It is an improved version of ant system. In ant system the disadvantage is that stagnation of search makes tour improvement impossible. When stagnation occurs, the trials on few edges grow so fast that ants always choose the same path again and again. So MMAS allows best ants to update the trails. In MMAS minimum and maximum trial strength are introduced explicitly and it is depends on the average path length. It also provides local search heuristic. There are two ways to allow an ant to perform local search, first is that to allow all ants to perform local search and second is to iterationbest.

So in MMAS ant system with local search provides an adaptive framework for the solution of optimization problems.

In virtual machine placement, many of the research focus on the single objective criterion. But in real-world single objective does not provide full optimization, so in recent real-world application multi-objective criterion is used for optimization of computing resources wastage and power consumption. Ant colony system algorithm used for this purpose.

\subsubsection{Ant Colony Algorithm for virtual machine} placement:

/* In itialization */

1. Set the value of parameter $\alpha, \mathrm{q}_{0}, \rho_{\mathrm{l}}, \rho_{\mathrm{g}}, \tau_{0}$, NA (nu mber of ants) and M (number of iteration).

2. Initialize Pareto set $\mathrm{P}$ as empty.

3. Initialize all pheromone values to $\tau_{0}$.

$/ *$ Iterative loop*/

4. Repeat

5. For $\mathrm{j}=1$ to NA do

$/ *$ construct a solution*/

6. Sort the server list PL in random order

7. Repeat

8. Introduce new server from server list PL

9. Repeat

10. For each remaining VM that can be packed in to the current server do

11. Calculate desirability of movement acc. to Eq. $\eta \mathrm{i}, \mathrm{j}=\eta \mathrm{i}, \mathrm{j}, 1+\eta \mathrm{i}, \mathrm{j}, 2$

12. Calculate probability of movement acc. to Eq.

$$
P^{k}{ }_{i, j}=\left\{\begin{array}{l}
\alpha \times \tau_{i, j}+(1-\alpha) \times \eta_{i, j} \\
u € \Omega_{k}(j)\left(\alpha \times \tau_{u}, j+(1-\alpha) \times \eta_{u, j}\right), i € \Omega_{k}(j)
\end{array}\right.
$$

0 ,

End For

13

$/ *$ choice of the VM to assign*/

14. Draw q

15. If $\mathrm{q}<=\mathrm{q}_{0}$ then

16. Exploitation

$17 . \quad$ Else

18. Exploration

$19 . \quad$ End If

/*Local pheromones updating*/

20. Apply local updating rule : $\quad \tau_{\mathrm{i}, \mathrm{j}}(\mathrm{t})=$ $\left(1-\rho_{\mathrm{l}}\right) \tau_{\mathrm{i}, \mathrm{j}}(\mathrm{t}-1)+\rho_{\mathrm{l}} \cdot \tau_{0}$

21. Until no remaining VM fits in the server anymore

22. Until all VM are placed

23. End For

/*evaluation*/

24. Calculate the value of two objective for each solution in current ant population

25. If a solution in current ant population is not dominated by any other solutions in the current population and non-dominated solutions in the Pareto set $\mathrm{P}$, this solution is added to the set $\mathrm{P}$.

/*global pheromones updating*/

26. For each non-dominated solutions in the Pareto set $\mathrm{P}$ do

27. Apply global updating rule:

$$
\tau_{i, j}(t)=\left(1-\rho_{g}\right) \tau_{i, j}(t-1)+\frac{\rho_{g} \cdot \lambda}{P^{\prime}(S)+W(S)}
$$

28. End For

29. Until the maximum number of iteration is reached

30. Return the Pareto set $\mathrm{P}$ 


\section{CONCLUSION}

The ant colony optimisation algorithm was extensively compared with the multi-objective genetic algorithm. The combined implementation of both the algorithms fetches a more optimal result. The proposed algorithm when tested with literature instances proved to be more efficient in VM placement thereby reducing the resource wastage and reducing the power consumption of the servers.

\section{REFERENCES}

[1]. Grit L, Irwin D, Yumerefendi A, Chase J. Virtual machine hosting for networked clusters: Building the foundations for autonomic orchestration. Proceedings of the 2nd International Workshop on Virtualization Technology in Distributed Computing; 2006; p. 1-7.

[2]. Y. Zhang, Z-1.Pei, J-h.Yang, Y-c. Liang, An Improved Ant Colony Optimization Algorithm Based on Route Optimization and Its Applications in Traveling Sales man Problem, IEEE 2007. 1-42441509-8.

[3]. N. B. Sariff and O. Buniyamin, "Ant Colony System for Robot Path Planning in Global Static Environment", Selected Topics In System Science And Simulation In Engineering, ISSN: 1792-507X, ISBN: 978-960-474-230-1, pp. 192-197.

[4]. M. Dorigo and M. Gambardella. Ant colonies for the travelling salesman problem. BioSystems 43 (1997) 73-81

[5]. Dorigo M, Gambardella L. Ant colony system: A cooperative learning approach to the traveling salesman problem. IEEE Trans Evol Comput. 1997; 1(1):53-66.

[6]. M. Dorigo, M. Birattari and T. Stutzle, "Ant Colony Optimization Artificial Ants as a Computational Intelligence Technique", Iridia - Technical Report Series: TR/IRIDIA/2006-023.

[7]. Ikeda M, Barolli R, Koyamac A, Durresi A, De Marco G, Iwashige J. Performance evaluation of an intelligent $\mathrm{CAC}$ and routing framework for multimedia applications in broadband networks. J Comput Syst Sci. 2006; 72(7):1183-200.

[8]. V. Maniezzo, A.Colorni, and M.Dorigo, "The ant system applied to the quadratic assignmentproblem, Tech Rep. IRIDIA/9428, 1994, UniversitéLibre de Bruxelles, Belgiu m 